\title{
Komplikationen bei fehlerhafter Versorgungsstrategie rheumatisch Erkrankter
}

\section{Complications in the Treatment of Rheumatoid Patients}

\author{
Autor \\ R. Gaulke
}

Institut

Sektion Obere Extremität, Fuß- und Rheumachirurgie, Klinik für Unfallchirurgie, Medizinische Hochschule

Hannover, Hannover

Schlüsselwörter

Versorgungsstrategie, Rheumatiker, Fallstrick, Komplikation

Key words

therapy regimes, rheumatoid arthritis, pitfall, complication

Bibliografie

DOI https://doi.org/10.1055/s-0042-116424

Online-Publikation: 29.9.2016

Akt Rheumatol 2018; 43: 37-42

(c) Georg Thieme Verlag KG Stuttgart · New York ISSN 0341-051X

Korrespondenzadresse

Prof. Dr. med. Ralph Gaulke

Klinik für Unfallchirurgie

Sektion Obere Extremität, Fuß- und Rheumachirurgie

Medizinische Hochschule Hannover

Carl-Neuberg-Straße 1

30625 Hannover

Tel.: + 49-511-532-2015

gaulke.ralph@mh-hannover.de

\section{ZUSAMMENFASSUNG}

Der orthopädische Rheumatologe muss als Generalist befähigt sein, Behandlungsstrategien zu entwickeln um den komplexen
Erkrankungsmustern seiner Patienten gerecht zu werden. Neben umfassender Kennnisse der konservativen und operativen Therapie muss er gute Kenntnisse der immunsuppressiven Therapie aufweisen, um keine iatrogenen Komplikationen zu induzieren. Die Grundprinzipien der orthopädisch-rheumatologischen Versorgungsstrategie beinhalten als oberstes Ziel den Erhalt oder die Wiederherstellung der Selbstständigkeit sowie die Schmerzbefreiung des Patienten. Nach der Schmerzbefreiung kommt der Gehfähigkeit des Patienten die höchste Priorität zu. Die Funktion der Hand, an der auch fortgeschrittene Fehlstellungen häufig lange kompensiert werden, spielt gleichfall eine große Rolle bei der Selbstversorgung. Da die Effekte einer zentralen Korrektur auf die peripheren Gelenke deutliche ausgeprägter ist als umgekehrt, sollte grundsätzlich von zentral nach peripher korrigiert werden. Die Missachtung dieser Prinzipien führt zu vermeidbaren Komplikationen, welche an Fallbeispielen dargestellt werden.

\section{ABSTRACT}

Being "all-rounders", orthopaedic rheumatologists have to develop complex treatment regimes to meet the needs of their polyarthritic patients. Profound knowledge of conservative, operative and immunosuppressive drug treatment is essential to avoid iatrogenic complications. The first aim in the treatment of rheumatoid patients is to alleviate pain and to keep patients self-supported, followed by restoring gait and the function of the hand. The correction of central deformities has a higher corrective effect on the peripheral joints than the reverse process. Therefore, correction should be central to peripheral. Ignoring this principle leads to avoidable complications, which will be illustrated in case studies.

\section{Einleitung}

In Zeiten der zunehmenden Spezialisierung in der Chirurgie des Bewegungsapparates kommt dem orthopädischen Rheumatologen als Generalisten eine Sonderstellung zu. Er muss in hohem Maße in der Lage sein, komplexe Erkrankungsmuster zu diagnostizieren, und die Bedeutung der Erkrankung einzelner Gelenke für die Funktion des ganzen Körpers abzuschätzen. Darüber hinaus muss er die Effekte eines sehr breiten Spektrums an konservativen und operativen Therapien auf den ganzen Bewegungsapparat kennen, um einen effektiven Behandlungsplan erstellen zu können. Daneben benötigt er profunde internistische Kenntnisse, welche sich nicht alleine auf die spezielle immunsuppressive medikamentöse Therapie des Rheumatikers beschränken. Dies begründet sich darauf, dass Rheumatiker nach langjährigem Krankheitsverlauf häufiger an Herz-Kreislauf- und Tumorerkrankungen aber auch an Diabetes mellitus und andere chronischen Krankheiten leiden.

Im Folgenden sollen die Therapiekonzepte in der orthopädischen Rheumatologie dargestellt werden. An Fallbeispielen sollen 
erläutert werden, zu welchen vermeidbaren iatrogenen Komplikationen eine Missachtung dieser Versorgungsstrategie führen kann.

\section{Prinzipien der Versorgungsstrategie}

Der Therapeut sieht häufig die Fehlstellung und die fehlende Funktion als Motivation dem Patienten eine operative Therapie anzubieten. Für den Patienten hingegen steht die Schmerzbefreiung immer an erster Stelle. Die eingeschränkte Funktion und kosmetische Aspekte führen den Patienten ebenfalls zum Arzt, stellen aber gegenüber dem Schmerz eine geringere Motivation dar, sich einer Operation zu unterziehen.

Die Grundlage für die Erarbeitung eines Therapiekonzeptes zur ganzheitlichen Versorgung bildet neben der Anamnese die Ganzkörperuntersuchung. Bei dieser werden alle Gelenke im Bezug auf Schwellung, Druck- und Translationsschmerz, Bewegungsausmaß, Stellung und Stabilität untersucht. Auch die Schwellungen der Sehnenscheiden und Schleimbeutel, sowie die Muskelfunktion und die Sensibilität werden dokumentiert.

Die Versorgungsstrategie richtet sich nach den Erfordernissen und Ansprüchen des Patienten und nicht alleine nach den klinischradiologischen Befunden. Eine Ausnahme bilden hierbei schmerzlose Veränderungen, die bei fehlender Versorgung zu Schäden führen. Diese gilt es durch eine Operation zu vermieden. Klassische Beispiele hierfür sind Tenosynovialitiden und schmerzfreie destru-

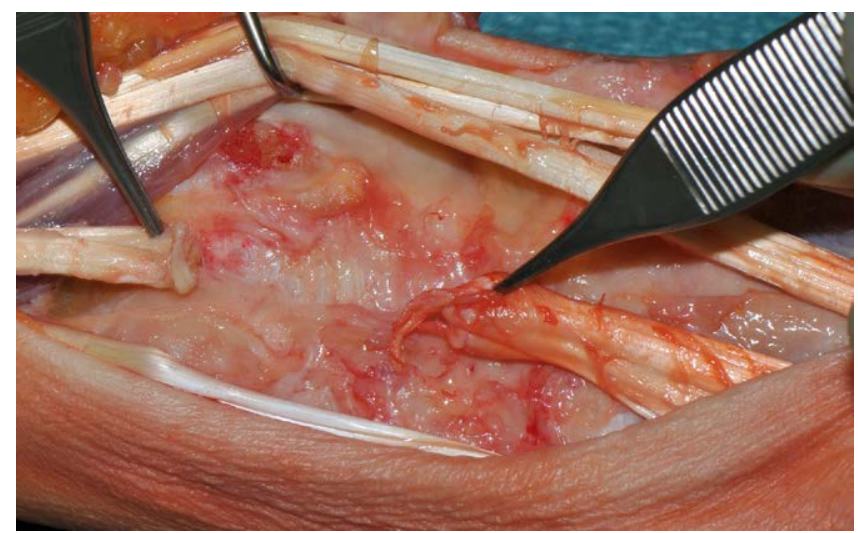

Abb. 1 Defektruptur der Extensor digitorum communis-Sehnen III-V über dem Ulnaköpfchen an der rechten Hand. Intraoperativer Befund nach Extensorentenosynovialektomie.

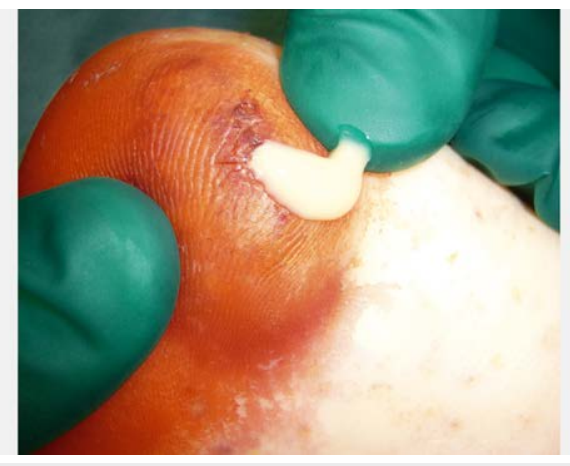

Abb. 2 Fistelnde Bursitis subkutanea achillae rechts. ierende Arthritiden mit und ohne Fehlstellung, welche unbehandelt im Verlauf zu Defektrupturen der Sehnen bzw. sekundären Arthrosen führen ( $\triangleright$ Abb. 1).

\section{Konservative Therapie}

Die medikamentöse Therapie stellt den zentralen Pfeiler zur Behandlung der Grunderkrankung dar. Sie hemmt die Entzündungsaktivität, birgt aber durch die Immunsuppression die Gefahr von Infektionen. Unter der Biologikatherapie können Infektionen so verschleiert werden, dass diese sowohl klinisch als auch laborchemisch unerkannt bleiben und zum Tode durch eine Sepsis führen können. Um septischen Komplikationen rechtzeitig erkennen und behandeln zu können, ist eine wiederholte gründliche Patientenaufklärung erforderlich und von ärztlicher Seite besondere Wachsamkeit geboten. So bedürfen unter wirksamer Basistherapie verbleibende oder neu auftretende Schwellung von Gelenken, Schleimbeuteln und Sehnenscheiden der diagnostischen Punktion zum Ausschluss einer Infektion ( A Abb. 2) [1-3].

Neben der medikamentösen Behandlung der Grunderkrankung sind die Injektionstherapie, die physikalische Therapie zur Erlernung der Gelenk-schonenden Ausübung von Tätigkeiten des täglichen Lebens und der Muskelkräftigung, die Hilfsmittelversorgung, die Orthetik und die orthopädische Schuhzurichtung und -versorgung wichtige Komponenten der konservativen Therapie. Diese sollten bei jedem Patienten in Betracht gezogen werden, der noch keine oder kompensierte Veränderungen zeigt. Auch bei Patienten, welche nicht operabel sind, kann mit der konservativen Therapie häufig eine Schmerzreduktion oder -befreiung erzielt werden und eine weitgehend selbstständige Lebensführung in vielen Fällen lange erhalten bleiben.

Die konservative Therapie vermeidet zwar die Risiken einer Operation, kann aber auch zu einer weiteren Einschränkung des Patienten führen. So führen orthopädische Stiefel ( $>$ Abb. 3) und aufwendige Orthesen häufig durch ihr Gewicht zu einer Mehrbelas-

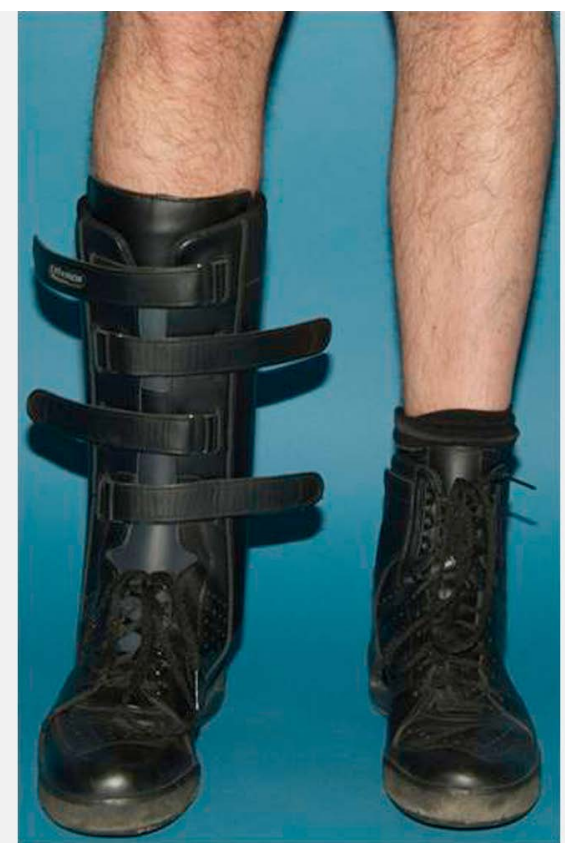

Abb. 3 Arthrodesenstiefel rechts. 
tung der übrigen arthritischen Gelenke und der Wirbelsäule und bedingen darüber hinaus eine schnelle Ermüdung der muskelschwachen multimorbiden Patienten.

Die intraartikuläre Injektionsbehandlung mit Kortikoiden kann einzelne aktive Gelenke unter wirksamer Basistherapie soweit beruhigen, dass die Arthritis danach unter der medikamentösen Therapie beruhigt bleibt. Besteht die Arthritis aber weiter fort, so sollte das Gelenk der Synovialektomie zugeführt werden, um den Schmerz zu nehmen und der Destruktion entgegenzuwirken. Tenosynovialitiden, welche sich unter suffizienter medikamentöser Therapie nicht zurückbilden, sollten gleichfalls zügig der operativen Therapie zugeführt werden, um Defektrupturen zuvorzukommen. Bei kleinen Gelenken kann alternativ zur operativen Synovialektomie eine Radiosynoviorthese (RSO) durchgeführt werden. An großen Gelenken sollte vor einer RSO die Dicke der Synovialitis sonografisch bestimmt werden. Überschreitet die Dicke der Synovialitis die Eindringtiefe des Isotops, wie dies an den großen Gelenken häufig der Fall ist, so sollte auf eine RSO verzichtet werden, da von den tiefen, nicht erreichten Synovialschichten eine hohe Rezidivgefahr ausgeht. In diesen Fällen hat sich das 2-zeitige Vorgehen mit der arthroskopischen oder offenen Synovialektomie und anschließender RSO nach Heilung der Kapselnarben bewährt, um die
Radikalität der Synovialektomie zu erhöhen und somit die Rezidivrate zu vermindern.

Ist die konservative Therapie ausgeschöpft, oder stellt diese z. B. durch schwere orthopädische Schuhe oder unbequeme Orthesen ihrerseits eine Beeinträchtigung des Patienten dar, oder drohen unter konservativer Therapie Folgeschäden, ist die operative Therapie unter Beachtung des Risikoprofils zu erwägen.

\section{Operative Therapie}

Vor der operativen Therapie muss ein Ganzkörperstatus erhoben werden. Die Biologika- bzw. Basistherapie sollte wenn möglich entsprechend der Empfehlungen der deutschen Gesellschaft für orthopädische Rheumatologie (DGORh) pausiert werden. Alle Fehlstellungen, Instabilitäten und Destruktionen werden evaluiert und mit den Beschwerden des Patienten abgeglichen. Die Versorgungsstrategie der Beine unterscheidet sich dabei wesentlich von der der oberen Extremitäten. Während die Stabilität an den belasteten Extremitäten das vorrangige Therapieziel darstellt, ist an der oberen Extremität die Beweglichkeit von größerer Bedeutung.

Bei paarig kontrakten Hüft- und Kniegelenken sollten beide Gelenke einzeitig oder 2-zeitig in einem Intervall von 3 Monaten versorgt werden, da sonst das kontrakte Gelenk das ersetzte wieder in die Kontraktur zwingt ( $\mathbf{A b b} \mathbf{4} \mathbf{4})$.

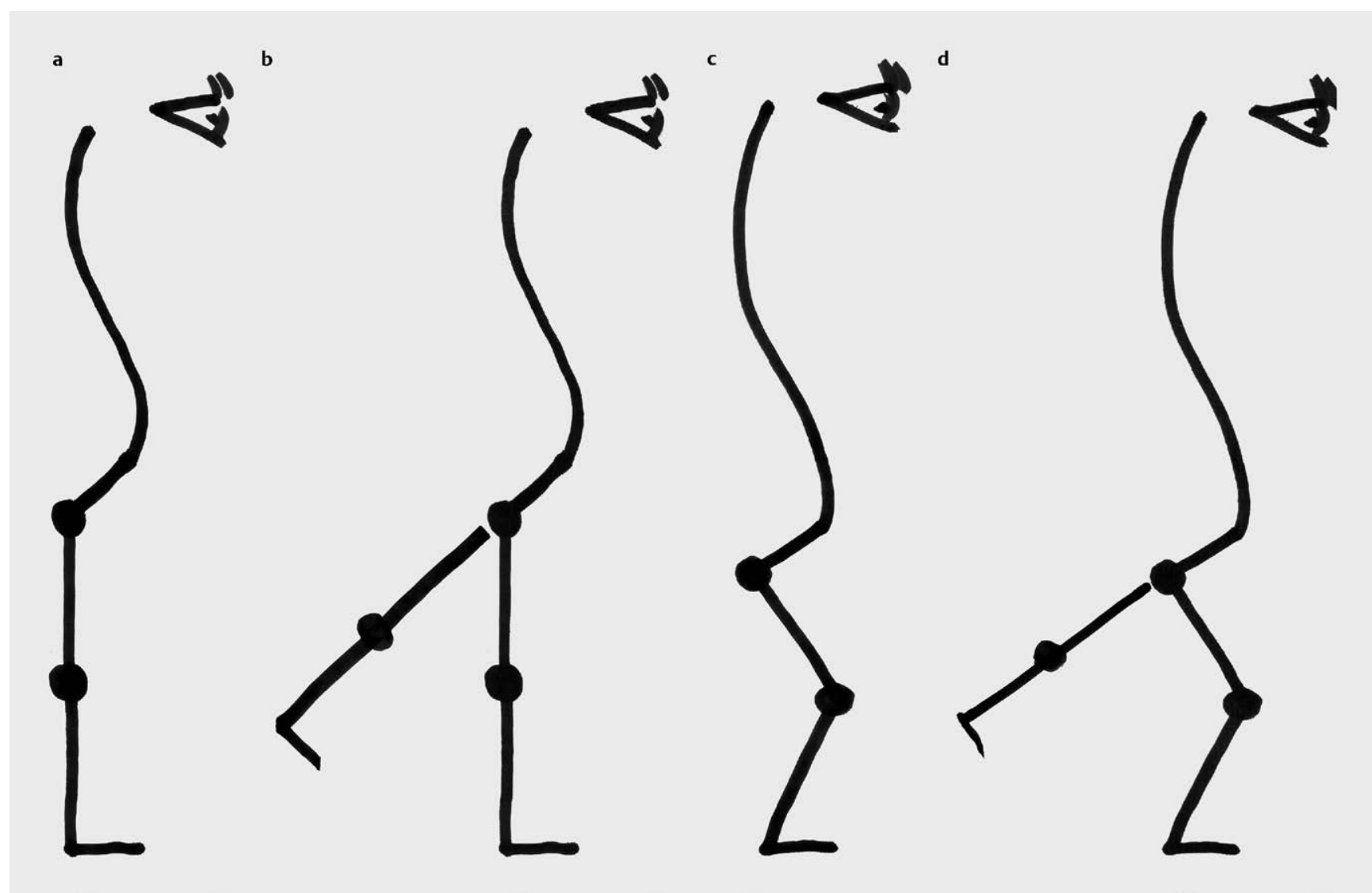

Abb. 4 a Die Hüftbeugekontraktur wird durch eine Hyperlordose kompensiert. b Zur Streckung der Hüfte, nach operativer Beseitigung der Beugekontraktur auf einer Seite mittels Endoprothese, müsste das operierte Bein, aufgrund der verbliebenen Kontraktur des gegenseitigen Hüftgelenkes, welche die Hyperlordose fixiert, um den Winkel der Kontraktur nach dorsal gestreckt werden. Da diese Bewegung in hohem Maße unphysiologisch ist, wird sie nicht durchgeführt und es tritt eine Rezidivkontraktur ein. c Bei Zunahme der Hüftbeugekontraktur wird nach Überschreiten der Kompensation durch die Hyperlordose das gleichseitige Kniegelenk gebeugt. d In diesem Falle müsste das Bein noch weiter nach hinten geführt werden, um die behandelte Hüfte zu strecken. 

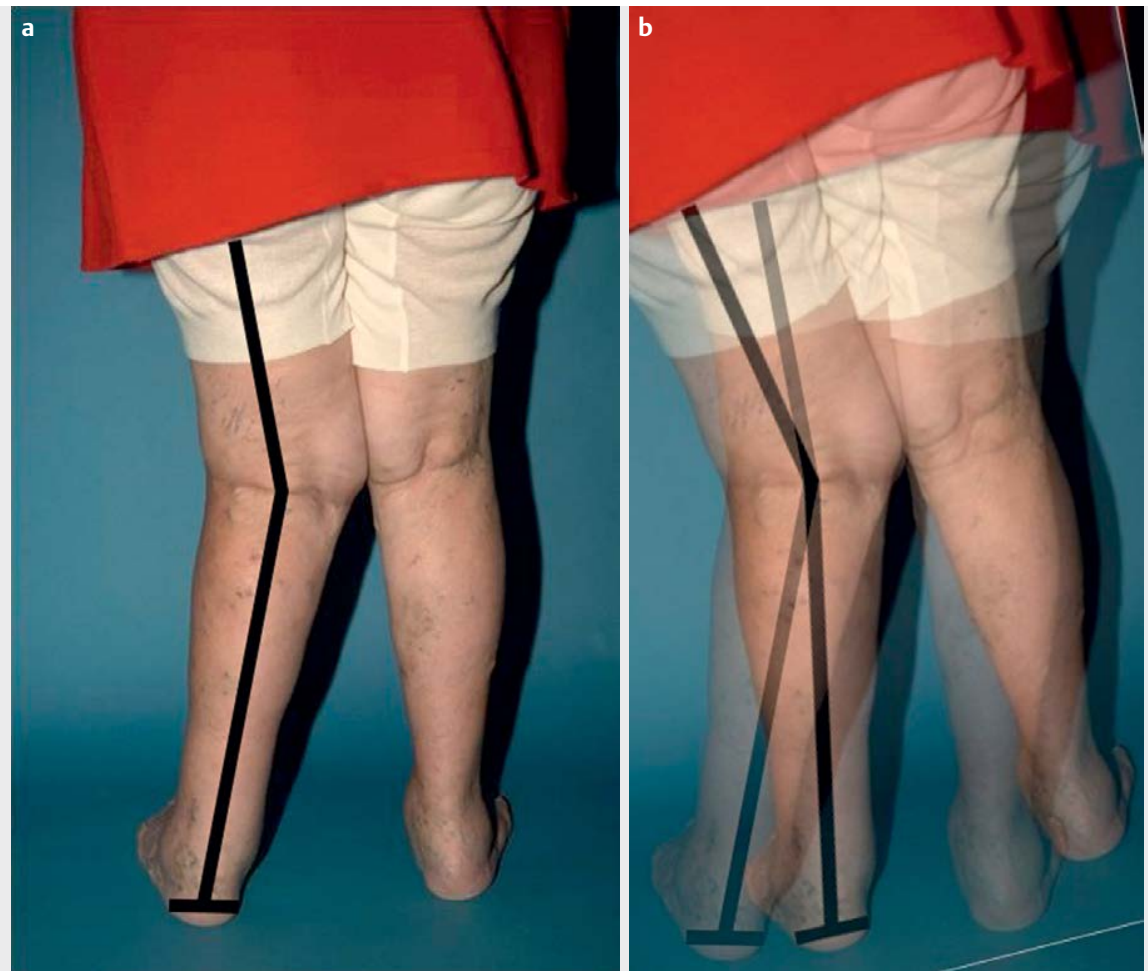

- Abb. 5 a Schmerzen über dem I. Strahl des li. Fußes bei Genu valgum. b Wird nun der Fuß in plantigrader Stellung korrigiert, so führt die sekundäre Beinachsenkorrektur, beispielsweise durch eine Knie-TEP, zu einer Supination des Fußes mit der Folge einer Überlastung der fibularen Strahlen. Die fibularen Schmerzen erfordern dann eine erneute Korrekturoperation zur plantigraden Ausrichtung des Fußes, welche bei Einhaltung der Therapieprinzipien nicht erforderlich gewesen wäre.

Die Beine müssen für den ergonomischen aufrechten Gang und zur Entlastung der Wirbelsäule gestreckt werden können. Kontrakturen verändern die Körperachse. Diese kann nur durch eine kompensatorische Fehlhaltung benachbarter Gelenke wieder ins Lot gebracht werden. Die benachbarten Gelenke werden durch die dauerhafte Fehlhaltung ihrerseits kontrakt. Beugekontrakturen der Hüfte führen zur kompensatorischen Hyperlordose der LWS und bei weiterem Fortschreiten zu Beugekontrakturen der Kniegelenke. Beugekontrakturen im Kniegelenk führen ihrerseits zu Beugekontrakturen der Hüften. Erweist sich die Hüftbeugekontraktur unter physiotherapeutischer Behandlung als Therapie-refraktär, sollte die Hüfte vor dem Kniegelenk ersetzt werden - auch wenn der Patient die stärksten Schmerzen im Kniegelenk angibt ( $\mathbf{A b b}$. 4).

In der Koronarebene besteht eine ähnliche Abhängigkeit. So wird ein Rückfuß valgus durch ein Genu valgum verstärkt und durch eine Genu varum vermindert. In diesem Fall muss vor einer plantigraden Einstellung des Fußes zunächst die Beinachse im Knie korrigiert werden. Wird zunächst der Fuß durch eine Rückfußarthrodese plantigrad eingestellt und dann das Genu valgum durch eine Knieprothese begradigt, so steht der Fuß danach in Supination und bedarf einer erneuten operativen Korrektur, die durch eine gute Versorgungsstrategie hätte vermieden werden können ( $\triangleright$ Abb. 5).

Am Fuß sollte die Korrektur des Rückfußes vor der Korrektur von Vorfußdeformitäten erfolgen, da die Vorfußbelastung maßgeblich von der Stellung des Rückfußes beeinflusst wird. So führt eine Varisierung des Rückfußes zu einer Vorfußsupination mit Mehrbelastung der fibularen Strahlen, während eine Valgisierung des Rückfußes zu einer Abflachung des Längsgewölbes und einer Pronation des Vorfußes mit Überlastung des I. Strahles führt. Die punktuelle Belastung führt bei dem häufig osteoporotischen Knochen zu schmerzhaften Ermüdungsfrakturen. Durch die bei Rheumatikern nicht seltene Polyneuropathie werden plantare Ulzerationen begünstigt, welche bei den immunsupprimierten Patienten im Falle einer schnellen Infektionsausbreitung zum Extremitätenverlust und/oder einer lebensbedrohlichen Sepsis führen können.

Bei der Vorfußkorrektur kann durch eine Rezentrierung des Metatarsale I-Köpfchens mittels Arthrodese des Tarsometatarsale I-Gelenkes (TMT I) häufig das Großzehengrundgelenk nach Synovialektomie und tibialer Kapselraffung erhalten werden. Erfolgt jedoch die Großzehengrundgelenk (MTPI)-Arthrodese bei ausgeprägtem Spreizfuß zuerst und wird sekundär eine TMT I-Arthrodese erforderlich, so muss meistens auch die Stellung der MTP I-Arthrodese korrigiert werden.

Die Resektion der Metatarsaleköpfchen II-V sollte bei rheumatischen Erkrankungen, wie von Hoffmann und Tillmann beschrieben von plantar erfolgen, da nur so eine effektive Resektion der plantaren Bursen und eine suffiziente plantare Kapsulodese erfolgen kann. Die plantare Narbe wird nicht belastet, da die Mittelfußknochenstümpfe proximal von dieser enden und verursacht daher keine Beschwerden.

An der oberen Extremität hingegen ist die Seitensymmetrie von deutlich geringerer Bedeutung. Im Gegensatz zur unteren Extremität werden Längendifferenzen kaum wahrgenommen. Endoprothetische Versorgungen von Schulter und Ellenbogen unterliegen keiner biomechanischen Notwendigkeit hinsichtlich der Reihenfolge der Versorgung. Gelenkdestruktionen an der oberen Extremität 
werden zudem aufgrund fehlender Gewichtsbelastung deutliche weniger als behindernd wahrgenommen. So können die Patienten selbst chronische Schulterluxationen und ausgeprägte Destruktionen des Ellenbogens lange kompensieren. Die operative Therapie sollte daher an diesen Lokalisationen nicht voreilig erfolgen. Ausnahmen bilden Nervenirritationen und Lähmungen bei instabilen Gelenken, welche einer dringenden Nervenentlastung bedürfen und progrediente Gelenkdestruktionen, die zu erheblichen Knochenverlusten führen können.

Die biomechanischen Aspekte der unteren Extremität finden ihre Entsprechung in der Strategie bei der Korrektur der Handdeformitäten [4]. Um dann die Sehnen an den Fingergrundgelenken zu rezentrieren ist es erforderlich, zunächst die Fehlstellung im Handgelenk zu korrigieren, um dann die Finger wirkungsvoll stabilisieren zu können. Hier sollte der Chirurg versiert in der Anwendung der zahlreichen Weichteileingriffe und Teilversteifungen sein, um bei der Wiederherstellung der Stabilität die größte mögliche Beweglichkeit zu erhalten $[5,6]$. Sollte sich die Handgelenkarthrodese nicht vermeiden lassen, so sollte diese in $0^{\circ}$ Stellung erfolgen, um bei beidseitiger Versteifung die selbstständige Körperpflege zu ermöglichen [7, 8]. Hier besteht die Gefahr, dass ein ausschließlich in der traumatischen Handchirurgie ausgebildeter Handchirurg die Handgelenkarthrodese beidseits in Extension durchführt und somit den Patienten zum Pflegefall operiert. Die Alternative der Versteifung eines Handgelenkes in leichter Extension und des kontralateralen in leichter Flexion ist gegenüber der beidseitigen Versteifung in $0^{\circ}$-Stellung kosmetisch ungünstiger und bietet nur in Ausnahmefällen funktionelle Vorteile. Bei Bewegungseinschränkungen in den Finger- und Ellenbogengelenken kann es erforderlich sein die Stellung der Handgelenkarthrodese individuell anzupassen. Es hat sich in diesen Fällen bewährt, vor der Arthrodese das Handgelenk im Unterarmgips solange in verschiedenen Positionen ruhigzustellen, bis die für den Patienten günstigste Stellung gefunden ist.

Auch an den Fingern ist die Korrektur der proximalen Fehlstellung in vielen Fällen von zentraler Bedeutung. So ist bei der Schwanenhalsdeformität die Rezentrierung der Fingergrundgelenke für die Wiederherstellung der Mittelgelenkbeugung notwendig [9]. Bei der Korrektur der Adduktionsstellung des Daumens kommt der Rezentrierung des Daumensattelgelenkes eine zentrale Bedeutung zu [10]. Bei der 90-90-Deformität des Daumens kann vor eintreten einer Kontraktur im Interphalangealgelenk dieses durch eine Daumengrundgelenkteno- oder -arthrodese in der Stellung korrigiert und dessen Funktion wieder hergestellt werden.

Diese Beispiele verdeutlichen die Gefahr der der Rheumatiker ausgesetzt ist, wenn er die einzelnen Gelenke durch mehrere Spezialisten versorgen lässt, welche ihre Therapie auf das betreffende Gelenk fokussieren ohne die Folgen für andere Körperregionen zu beachten. Da diese in der Regel keinen der Gesamtsituation adäquaten Therapieplan erstellen wird der Patient häufig von dem Spezialisten zuerst operiert, dessen Sprechstunde er zuerst aufsucht. In diesem Falle folgt die Reihenfolge der Versorgung dem Zufall an Stelle einer Strategie. Dies führt in vielen Fällen zu Revisionseingriffen, welche es im Sinne des Patienten unbedingt zu vermieden gilt.

\section{Triage des operativen Vorgehens}

Da sich die Dringlichkeit operativer Eingriffe, aufgrund der Dynamik der rheumatischen Erkrankungen, mit der Zeit verschieben kann, ist es unbedingt erforderlich, die Versorgungsstrategie unmittelbar präoperativ zu überprüfen und die einzelnen Dringlichkeiten zu gewichten (Triage).

Eine Op-Indikation mit höchster Dringlichkeit (Notfall: Op sofort) besteht beim septischen Gelenk, septischer Sehnenscheidenentzündung, Abzess und Phlegmone, zentralen und peripheren Lähmungen, akuter Blutung, offener Fraktur, akuter Luxation und Kompartmentsyndrom.

Eine Op-Indikation mit hoher Dringlichkeit (Op innerhalb einer Woche) besteht bei persistierender Tenosynovialitis über 6 Wochen trotz konservativer Therapie unter suffizienter Basistherapie, stark progredienter destruierender Arthritis, spontaner Sehnenruptur, sehr starken unbeherrschbaren Schmerzen, geschlossener Fraktur.

Eine Op-Indikation mit niedriger Dringlichkeit (Op innerhalb von 4 Wochen) besteht bei destruierender Arthritis ohne Option das Gelenk noch retten zu können aber mit fortschreitendem Knochenverlust, der u. U. die Verwendung einer größeren Prothese erforderlich macht. Auch progrediente Fehlstellungen an Händen und Füßen mit zunehmenden Weichteilkontrakturen, die eine Rezentrierung erschweren, gehören in diese Gruppe.

Eine Op-Indikation ohne Dringlichkeit (Op-Zeitpunkt vom Patienten frei wählbar) bestehen für eine Arthrodese und Endoprothese bei sekundärer Arthrose mit stabilen Knochenverhältnissen ohne grobe Fehlstellungen, welche nur mäßige Beschwerden bereitet.

\section{Zusammenfassung}

Die Spezialisierung der Operateure auf einzelne Körperregionen oder gar einzelne Gelenke wird von den Kostenträgern mit dem Argument der Qualitätssteigerung unterstützt. Dieser Trend spiegelt sich in der Außendarstellung vieler Kliniken durch eine inflationäre Zunahme von Zentren für einzelne Therapie wieder. Für Patienten mit Systemerkrankungen des Bewegungsapparates, welche einer ganzheitlichen Betrachtung bedürfen, ist diese Entwicklung jedoch gefährlich. Vergleichbar ist die Therapie der Rheumatiker in ihrer Ganzheitlichkeit mit der Therapie Polytraumatisierter, welche jedoch in der Regel einer wesentlich höheren Dringlichkeit unterliegt. Wohl aus diesem Grunde ist am Bewegungsapparat neben dem Unfallchirurgen und dem Kinderorthopäden der orthopädische Rheumatologe einer der letzten Generalisten geblieben.

Die Versorgung von Patienten mit Erkrankungen des rheumatischen Formenkreises erfordert ein sehr breites operatives Spektrum und sehr gute Kenntnisse um die Pathobiomechanik arthritischer Gelenke. Daneben ist die interdisziplinäre Zusammenarbeit mit dem internistischen Rheumotologen unabdingbar, um das $\mathrm{Ne}$ benwirkungsprofil der weiterhin in großer Zahl auf den Markt drängenden Biologica im Umfeld der operativen Therapie beurteilen zu können. Nur in vertrauensvollem Umgang mit den internistischen Rheumatologen kann sicher gestellt werden, dass das therapeutische Fenster zum Gelenk- und Sehnenerhalt genutzt und unnötige Operationen vermieden werden. 


\section{Interessenkonflikt}

Nein.

\section{Literatur}

[1] Gaulke R. Unterarmphlegmone unter Anakinra. Z Rheumatol 2003; 62: 566-569

[2] Garcia-Arias M, Balsa A, Mola EM. Septic arthritis. Pract Res Clin Rheumatol 2011; 25: 407-421

[3] Wang DA, Tambyah PA. Septicarthritis in immunocompetent and immunosuppressed hosts. Best Pract Res Clin Rheumatol 2015; 29 : 275-289

[4] Pahle JA, Raunio P. The influence of wrist position on finger deviation in the rheumatoid hand. A clinical and radiological study. J Bone Joint Surg [Br] 1969; 51: 664-676
[5] Gaulke R, O'Loughlin PF, Probst C et al. Comparative biomechanical analysis of two techniques of radiolunate fusion: Shapiro staples vs. plate and oblique screw. Technol Health Care 2010; 18: 111-121

[6] Gaulke R, Oszwald M, Liodakis E et al. Comparative biomechanical analysis of two techniques of radiolunate plate-fusion: oblique screw vs. parallel screws. Technol Health Care 2012; 20: 225-232

[7] Rayan GM, Brentlinger A, Purnell D et al. Functional assessment of bilateral wrist arthrodesis. J Hand Surg [Am] 1987; 12: 1020-1024

[8] Gaulke R, Krettek C. Die Handgelenkarthrodese beim Rheumatiker. Orthopäde 2007; 36: 729-734

[9] Ramsauer T, Hilker A. Schwanenhals- und Knopflochdeformität der Langfinger. In: Rehart S, Sell S, (Hrsg.). Expertise Orthopädische Rheumatologie. Stuttgart: Thieme; 2016

[10] Heniger M, Rehart S. Daumen und Langfinger. In: Rehart S, Sell S, (Hrsg.). Expertise Orthopädische Rheumatologie. Stuttgart: Thieme; 2016 